Article

Discoveries Section

\title{
Stepwise functional evolution in a fungal sugar transporter family
}

Carla Gonçalves ${ }^{\mathrm{a}}$, Marco A. Coelho ${ }^{\mathrm{a}}$ Madalena Salema-Ooma, and Paula Gonçalves ${ }^{\mathrm{a},{ }^{\mathrm{a}} \text {, }}$

aUCIBIO-REQUIMTE, Departamento de Ciências da Vida, Faculdade de Ciências e

Tecnologia, Universidade NOVA de Lisboa, 2829-516, Caparica, Portugal

bInstituto Superior Ciências da Saúde Egas Moniz, Centro de Investigação Interdisciplinar Egas

Moniz (CiiEM), 2829-511, Caparica, Portugal

\section{*Corresponding Author:}

Paula Gonçalves

\section{UCIBIO-REQUIMTE}

Departamento de Ciências da Vida

Faculdade de Ciências e Tecnologia

Universidade Nova de Lisboa

2829-516, Caparica, Portugal

Tel: (+351) 212948530

Email:pmz@fct.unl.pt 


\begin{abstract}
Sugar transport is of the utmost importance for most cells and is important to a wide range of applied fields. However, despite the straightforward in silico assignment of many novel transporters, including sugar porters, to existing families, their exact biological role and evolutionary trajectory often remain unclear, mainly because biochemical characterization of membrane proteins is inherently challenging, but also owing to their uncommonly turbulent evolutionary histories. In addition, many important shifts in membrane carrier function are apparently ancient, which further limits our ability to reconstruct evolutionary trajectories in a reliable manner.

Here we circumvented some of these obstacles by examining the relatively recent emergence of a unique family of fungal sugar facilitators, related to drug antiporters. The former transporters, named Ffz, were previously shown to be required for fructophilic metabolism in yeasts. We first exploited the wealth of fungal genomic data available to define a comprehensive but welldelimited family of Ffz-like transporters, showing that they are only present in Dikarya. Subsequently, a combination of phylogenetic analyses and in vivo functional characterization was used to retrace important changes in function, while highlighting the evolutionary events that are most likely to have determined extant distribution of the gene, such as horizontal gene transfers (HGTs). One such HGT event is proposed to have set the stage for the onset of fructophilic metabolism in yeasts, a trait that according to our results may be the metabolic hallmark of approximately one hundred yeast species that thrive in sugar rich environments.
\end{abstract}




\section{Introduction}

Sugar transport is a biological process of paramount importance, since for a wide variety of organisms in all kingdoms of life, sugars (mono and di-saccharides) are favorite carbon and energy sources. Partitioning and distribution of sugars is accordingly found to be disturbed in various important animal and plant diseases. For example, one important biochemical hallmark of cancer cells is their increased rates of glucose uptake (Ganapathy-Kanniappan and Geschwind 2013) and some bacterial plant pathogens are able to increase sugar levels in their vicinity by inducing the expression of particular sugar transporter genes in the host plant (Streubel et al. 2013). Moreover, industrial substrates for microbial growth are often sugar-rich and research on microbial biotechnology based processes, such as beer and bioethanol production, revealed over the past few decades several examples of the high impact of the sugar transport step on overall fermentation performances (Reznicek et al. 2015; Vidgren et al. 2010; Young et al. 2012). This was also patent when domesticated microbes were genetically dissected and compared to their "wild counterparts" (Libkind et al. 2011; Perez-Ortin et al. 2002). Hence, an improved understanding of sugar transport is pertinent to a wide variety of areas of applied interest, from human health to agricultural crop yield and biotechnology. In spite of this, the elucidation of functional and structural aspects of integral membrane proteins, remains inherently challenging, resulting in a considerable deficit in available biochemical data for in silico predicted transporter proteins, when compared to their soluble metabolic enzyme counterparts (Reddy et al. 2012).

While approximately 800 families of cellular solute transporters have been identified, based on functional and phylogenetic evidence (Saier et al. 2014), most eukaryotic sugar carriers belong to the largest superfamily of transporters, the so-called Major Facilitator Superfamily (MFS) (Pao et al. 1998). This superfamily is ubiquitously distributed in the biosphere, and is characterized on the one hand by considerable structural conservation and, on the other hand, by an astounding diversity of substrates and of modes of operation (uniport, symport and antiport) (Reddy, et al. 2012). The few sugar transporters that do not belong to MFS are a well-studied human glucose transporter (SGLT) placed in the solute:sodium symporter family (Wright et al. 2011) and a novel type of carrier generically dubbed SWEET (or SemiSWEET) that was recently discovered in plants, animals and bacteria and fits into the Transporter-Opsin-G-protein coupled receptor (TOG) superfamily (Feng and Frommer 2015). In fungi, all sugar transporters characterized so far are included in the Sugar Porter (SP) family within MFS, with the notable exception of those belonging to the Ffz-like family which are the main subject of this work (Leandro et al. 2011) and were rather included in a different MFS family formed by Drug: $\mathrm{H}^{+}$ antiporters (DHA1 family). 
Ffz transporters have so far been found only in a limited number of fungal species (Cabral et al. 2015; Leandro, et al. 2011; Lee et al. 2014; Pina et al. 2004). In vivo biochemical characterization showed that they are usually high capacity and low affinity uniporters, specific for fructose (Leandro, et al. 2011; Lee, et al. 2014; Pina, et al. 2004), while genetic analyses showed that Ffz1 seems to be a pre-requisite for fructophily in at least one yeast species (Leandro et al. 2014). Fructophily, defined as the preference for fructose over glucose as carbon and energy source, is a metabolic trait well characterized so far in a few yeast species found in high sugar environments (Pina, et al. 2004; Tofalo et al. 2009) and in some bacteria (Endo et al. 2009; Endo and Salminen 2013). The singularity of the evolutionary origin of the Ffz family of transporters and its consistent association with fructophily in yeast taxa belonging to widely different lineages but inhabiting similar habitats (Sousa-Dias et al. 1996; Yu et al. 2006), suggest an evolutionary path punctuated by profound but relatively recent functional change and opens the possibility to establish a link between functional evolution and certain ecological traits. In fact, the organization of transporter families within the MFS Superfamily shows a general agreement between phylogenetic relatedness and the type of substrate accepted by the transporter. For example, the Most Recent Common Ancestor (MRCA) of the Sugar Porter family probably goes far back in evolutionary history, as this family comprises members spanning the diversity of life, from human to bacteria, all accepting only sugars or related compounds as substrates. Hence, the emergence of a sugar transporter "sub-family" within a Drug: $\mathrm{H}^{+}$antiporter family is likely to be comparatively a very recent event, possibly related to selective pressures associated to the adaptation of specific fungal lineages to new environments or lifestyles. In addition, within the Ffz family itself, some functional differences have been noted prior to this study, since the Ffz2 type of transporter accepts both glucose and fructose as substrates (Leandro, et al. 2011) contrary to the first identified member of the family, Ffz1, reported to accept only fructose (Pina, et al. 2004). All these considerations led us to regard the Ffz family as an excellent model in which to examine adaptive evolution of transporters and possibly to establish a relation between sugar transport and organismal ecology. As a consequence, we set out to explore the wealth of genomic data available for fungi to illuminate the evolutionary origin and history of the Ffz sugar transporter family.

Elucidation of the molecular evolution of sugar transporters is often complicated by redundancy and particularly rapid evolution (Brown et al. 2010; Lin and Li 2011). An emblematic example of this is the small genome of the model yeast Saccharomyces cerevisiae that encodes at least 17 hexose uniporters, named HXT. Biochemical characterization revealed similar substrate specificities for many of the Hxt transporters but clear differences in the affinity for glucose (Diderich et al. 2001; Reifenberger et al. 1997). However, provided that expression levels are appropriate, most $H X T$ genes seem to be able to support growth of $S$. cerevisiae on glucose, fructose and mannose (Diderich, et al. 2001; Reifenberger, et al. 1997). While retention of this 
plethora of partially redundant genes in the S. cerevisiae genome probably reflects the importance of sugar transport in the life style of this yeast, possibly functioning as a safety net, the presence of genes encoding at least one high and one low affinity transporter for the same nutrient is a common theme in fungi (Horak 2013) and may have an important role in enabling the cells to adjust rapidly to changing conditions, as elegantly shown for two S. cerevisiae transporters (Levy et al. 2011).

As judged from available genomic data, transporter gene families frequently undergo expansions and losses (Lin and Li 2011) and are also among the fungal genes that are most often involved in Horizontal Gene Transfers (HGT) (Coelho et al. 2013; Marsit et al. 2015). Accordingly, they are often located in highly variable genomic regions like the subtelomeres (Bergstrom et al. 2014; Brown, et al. 2010; Dias and Sa-Correia 2013). Because they constitute a favorable environment for loss and acquisition of genes, subtelomeres are also characterized by poorly conserved synteny (Kellis et al. 2003), complicating the reliable elucidation of orthology between genes, and consequently, the clarification of their evolutionary history with a satisfactory degree of certainty. Interestingly, the apparent plasticity of transporter gene evolution revealed by comparative genomics is generally recapitulated in laboratory evolution experiments, where adjustments in the transport step are among the first responses to strong selective pressures related to nutrient availability, including changes in expression (Kvitek and Sherlock 2011) and gene fusions (Brown et al. 1998) in addition to gene copy number variation (CNVs) (Gresham et al. 2008). All this tends to blur evolutionary footprints of very old events like the formation of substrate specialized MFS families. However, it is possible to learn much about such events by examining similar occurrences that took place at a more recent point in time, like seems to be the case for the origin of the Ffz family of sugar transporters.

In this study we confirm that Ffz-like sugar transporters are indeed found only in fungi and that they probably arose early in the evolution of Dikarya, because they are present both in the Ascomycota and the Basidiomycota, but not in early-branching fungal lineages. Interestingly, the biochemical characterization of several novel members of the family allowed us to reconstitute several functional transitions in the evolutionary history of the Ffz family. Our work also suggests that Ffz1 associated fructophily is likely to have evolved in an early-diverging yeast lineage encompassing currently more than 80 species, mainly associated with the fructose rich floricolous environment, after the horizontal acquisition of an Ffz1 like gene from a filamentous ascomycete (Pezizomycotina).

\section{Results}

\section{Close homologs of Ffz are present only in Dikarya}

Ffz transporters were included in the DHA1 family of drug: $\mathrm{H}^{+}$antiporters that comprises bacterial 
and animal proteins, in addition to fungal transporters (Saier, et al. 2014). To ascertain if Ffz-like transporters could be found outside the fungal kingdom, initial BLAST search analyses in publicly available databases were performed using the Ffz1 protein sequences from Zygosaccharomyces bailii as query. These analyses showed that only fungal proteins were retrieved up to an $E$-value of $1 e-08$, at which point members of the bacterial drug resistance transporter family $\mathrm{Bcr} / \mathrm{CflA}$ (Bentley et al. 1993) start to be recovered. The same set of bacterial proteins were retrieved as the closest Ffz1 relatives when fungal genomes were excluded from the search, protist and algal lineages being notably absent. The $E$-values obtained for some fungal DHA1 family members in these searches were as low as $1 e-85$, suggesting strong sequence similarity with Ffz1 and using an $E$-value cut-off of $1 e-40$ resulted in the sole retrieval of fungal DHA1 family members. Hence, we concluded that close Ffzl homologs could only be found in fungi. Finally, in order to get a first insight in the phylogenetic relationships between previously characterized Ffz transporters and the homologs retrieved in our query, including functionally characterized members of the DHA1 transporter family, we chose an E-value cut-off of $1 e-60$ that recovers a total of 1774 proteins. From these, 476 sequences representing all main fungal lineages, were chosen to construct a Maximum Likelihood (ML) phylogeny (supplementary fig. S1). In this tree, the deepest branches define two clades (A and B in supplementary fig. S1), one of which delimits the DHA1 family (clade A) and encompasses several functionally characterized transporters (Alarco et al. 1997; Barker et al. 2003; Calabrese et al. 2000; Tomitori et al. 2001; Wirsching et al. 2001). The other clade (B) comprises a large number of uncharacterized transporters, as well as all the Ffz-like transporters known so far, namely the Ffz1 and Ffz2 proteins from Zygosaccharomyces species (Leandro, et al. 2011) and Ffz1 transporters recently described in the yeast Candida magnoliae (Lee, et al. 2014) and in a mold, Aspergillus brasiliensis (Cabral \& Leandro, personal communication). Within clade B, known Ffz homologs cluster together with other, so far uncharacterized, proteins in one monophyletic group $(\mathrm{C}$, in supplementary fig. $\mathrm{S} 1)$, which we tentatively considered could represent a Ffz-like sugar transporter cluster. Sister clade D (supplementary fig. S1) consists entirely of uncharacterized proteins.

\section{A broad specificity Ffz-like hexose transporter in the Basidiomycota}

So far, Ffz-like fructose transporters were identified and characterized only in the Ascomycota. However, the Ffz-like transporter cluster (clade C, supplementary fig. S1) includes proteins from the three main lineages in the Basidiomycota and the Ascomycota (except for the Taphrinomycotina within the Ascomycota). To ascertain whether other proteins in this lineage, namely those originating from the Basidiomycota, function as sugar transporters, we undertook the functional characterization of a putative Ffz homolog uncovered by our survey in Ustilago maydis, a basidiomycete fungal pathogen of maize, causing corn smut (Gold et al. 1997). To this end, we expressed transporter UM03908 from U. maydis included in clade C in a S. cerevisiae 
strain devoid of all hexose transporters and thus unable to grow on hexoses (henceforth referred to as $h x t$-null) (Wieczorke et al. 1999). The results depicted in fig. 1 show that the U. maydis transporter complements growth of the hxt-null strain on glucose, galactose and mannose. Interestingly, complementation of growth on fructose is hardly noticeable on solid medium and it is not detected in shake flask culture, showing that the U. maydis $\mathrm{Ffz}$ homolog is indeed a sugar transporter, but with a completely distinct substrate preference from Ffz transporters characterized so far. We subsequently expressed in the hxt-null strain three additional U. maydis transporters (UM02585, UM05393 and UM05981) included in the sister clade D that consists entirely of uncharacterized proteins. All three failed to complement growth on hexoses, suggesting that they have a distinct function (fig. 1). These results, together with the fact that none of the early-derived fungal lineages (e.g. Mucoromycotina) are represented in clade C (see Table S2 for a list of all the lineages surveyed) suggest that clade C (supplementary fig. S1) may represent a Ffz-like sugar transporter cluster with its origin in the MRCA of the Dikarya, before the separation between Ascomycota and Basidiomycota. Our survey of a total of 606 Dikarya genomes uncovered 187 transporters obeying the criteria defining clade $\mathrm{C}$ ( $E$-value and sequence identity thresholds in BLAST searches), which will henceforth be referred to as Ffz-like transporters.

\section{A close up on the distribution of Ffz homologs in Dikarya}

In order to get some insight into the evolutionary mechanisms that determined the extant phylogenetic distribution of FFZ-like genes in Dikarya, we first used available genome data to estimate the pervasiveness of the gene in the main lineages of Dikarya. As graphically depicted in the insert of fig. 2 and listed at species level in Table S2, FFZ-like genes do not seem to be evenly distributed. Although sampling is presently too disproportionate between the various lineages to allow firm conclusions to be drawn, so far the gene family seems to be relatively more abundant in the Ustilaginomycotina (Basidiomycota) and the Pezizomycotina (Ascomycota). It is absent from the Taphrinomycotina (Ascomycota), and it is very scantily represented in the remaining lineages, particularly in the relatively well-sampled Agaricomycotina (Basidiomycota). In the Saccharomycotina, where the gene was first uncovered it is also notably rare. To examine this distribution using a detailed phylogenetic analysis, we next plotted the presence/absence of FFZ-like genes onto a phylogenetic tree constructed using concatenated sequences of six RNA polymerase subunits, which recovers the expected phylogenetic relationships between the represented taxa (Parrent et al. 2009). The species included in the tree (fig. 2) were selected to represent all orders in the Pezizomycotina and all families in the Saccharomycotina for which more than three genome sequences were available (last updated in February 2015). The Pezizomycotina and the Saccharomycotina were singled out for this more detailed analysis because, according to the results described in the previous section, Ffz-like 
transporters mediating fructose uptake, i.e. true functional homologs of yeast Ffz transporters, seem to be circumscribed to these two lineages. Since Ffz1 is strongly linked to fructophily but very few genomes were available in lineages containing fructophilic yeasts (only those of $Z$. rouxii and $Z$. baillii), we obtained draft genome sequences from four additional fructophilic species, namely Zygosaccharomyces kombuchaensis, Starmerella bacillaris, Starmerella bombicola and Candida magnoliae. Starmerella bacillaris (syn. Candida zemplinina) is a strongly fructophilic species usually associated with the oenological environment (Englezos et al. 2015; Zott et al. 2008) and the other three species have been recently shown to be fructophilic under some conditions (Cabral, et al. 2015; Yu, et al. 2006). We also obtained the draft genome sequence of Wickerhamiella domercqiae which is part of a sister lineage of the clade formed by the C. magnoliae and Starmerella, a species with hitherto unknown carbon source preferences.

Notably, at least one FFZ-like gene was found in each of the five draft genomes. In $S$. bacillaris, two distinct genes were found at a distance of approximately $4 \mathrm{~kb}$ from each other (supplementary fig. S3) and were both related to FFZ1 genes from Zygosaccharomyces. Given the strong association of fructophily with the presence of this type of transporter, the finding of one FFZ1-like gene in the genome of $W$. domercqiae suggested it might be fructophilic, prompting us to evaluate the sugar preference profiles of this and two additional Wickerhamiella species, W. cacticola and W. australiensis. The results, depicted in fig. 3, showed that all three Wickerhamiella species tested were fructophilic to various degrees, since they consumed fructose first when cultivated in medium containing high concentrations of both glucose and fructose. This broadens considerably the phylogenetic range of the earliest-derived fructophilic yeast clade, since the Wickerhamiella and Starmerella lineages together include a large and increasing number of species, presently more than 80 (Lachance 2011; Lachance and Kurtzman 2011), suggesting that fructophily may be the hallmark of the metabolism of a much larger number of species than assumed thus far. This early-branching Saccharomycotina clade that includes fructophilic yeasts will be henceforth referred to as the Wickerhamiella/Starmerella (W/S) clade.

Finally, in the genome of Z. kombuchaensis, which belongs to the other yeast lineage consisting almost entirely of fructophilic species, and overlapping entirely with the Zygosaccharomyces genus, we found one FFZ1-like gene (the type that accepts only fructose as substrate) and an additional gene that presented higher sequence similarity with genes encoding Ffz2-like glucose/fructose transporters in Z. rouxiii (Leandro, et al. 2011) and Z. baillii (Pina, et al. 2004). This was the only new FFZ2-like gene uncovered in the present survey, reinforcing the notion that this type of gene exists only in the Zygosaccharomyces genus.

In the Pezizomycotina, Ffz-like transporter proteins were encoded in the 12 of the 20 orders surveyed (fig. 2 and Table S2). The proportion of Ffz-harboring and Ffz-lacking species was highly variable among the 12 orders where the gene was identified, which may be partly due to skewed sampling of the various taxa [the number of genomes examined varies between three 
(Erysiphales and Xylonomycetales) and 57 (Hypocreales)]. Of the two most abundantly and similarly sampled orders, the Eurotiales ( 53 species examined) and the Hypocreales ( 57 species examined), the gene is pervasive in the former and rarely found in the latter. These differences in distribution do not necessarily reflect phylogenetic distances since in the sister lineage of the Eurotiales (Onygenales), Ffz1 homologs were found to be absent in all but one of the 22 species sampled (see Table S2).

\section{Ffz1 phylogeny vs species phylogeny}

The paucity and patchy distribution of Ffz genes in both the Pezizomycotina and the Saccharomycotina raised the question of whether multiple duplications and losses in the various lineages were likely to be solely responsible for extant distribution of the gene, since patchy distribution is considered to be suggestive of horizontal gene transfers (Fitzpatrick 2012). To clarify which events were mainly responsible for shaping evolutionary history of the Ffz-like family, we carefully examined the phylogenetic relationships between the Ffz homologs presently uncovered. The ML phylogenetic tree shown in supplementary fig. S2 is suggestive of a complex pattern of ancient and more recent duplications and losses, of which the polyphyly of the Dothideomycetes is a good example, but is evident in many other instances where the Ffz ML tree does not recover the expected phylogenetic relationships between taxa. Notably, the first node within the Ffz-like family defines two clades, one containing Ffz proteins from all lineages in Dikarya and the other including only Ffz homologs from Basidiomycota. A few basidiomycete species have representatives in both clades, suggesting the occurrence of an ancient duplication event with loss of one of the paralogs in most species. One paralog was probably lost very early in the evolution of the Ascomycota because it is absent from all extant species examined in this phylum (supplementary fig. S2).

Other noteworthy unconformities with the expected species phylogeny were uncovered, namely involving the yeast Ffz homologs and those from the Eurotiales (Pezizomycotina). We subsequently focused our analysis mainly on aspects pertaining to the reconstruction of the evolutionary history of Saccharomycotina and Eurotiales (Pezizomycotina) homologs, where all Ffz transporters that have been characterized so far are included. To investigate this in detail, a ML phylogeny of all the species in the Saccharomycotina and the Eurotiales was firstly reconstructed using concatenated sequences of RNA polymerase subunits recovering, likewise, the expected topology for the species included (fig. 4A). Next, a ML phylogenetic tree reconstructed using only Ffz homologs from the Saccharomycotina and the Eurotiales (fig. 4B) confirmed two noteworthy discrepancies with the species tree already observed in the expanded ML phylogenetic tree depicted in Supplementary fig. S2. Firstly, the Ffz homologs from the 
Saccharomycotina cluster with the transporters from the two Monascus species (Eurotiales), instead of forming a separate cluster. This observation suggests that the gene may have been absent in the most recent common ancestor (MRCA) of the Saccharomycotina, being instead captured by the MRCA of the W/S clade through HGT from a species close to Monascus (Event A; fig. 4B). This is consistent with the partially conserved synteny around the FFZ locus in the three species in the Starmerella clade (Supplementary fig. S3), since synteny with Wickerhamiella is not expected given the phylogenetic distance from Starmerella. Additional support to this event is also provided by the scarcity of Ffz homologs observed in yeasts, including their absence in the earliest derived lineages represented in fig. 4A by the species Lipomyces starkeyi and Tortispora caseinolytica. Secondly, the Wickerhamiella Ffz homolog did not associate with the Starmerella branch of the tree in the Ffz phylogeny, as might be expected according to the species phylogeny, but clustered rather with the Zygosaccharomyces Ffz clade. We interpret this observation as being suggestive of another HGT event (Event B, fig. 4B), consisting in the acquisition of an FFZ gene by the MRCA of Zygosaccharomyces from a donor in the Wickerhamiella lineage. This is supported by synteny analysis of the FFZ1 locus in the three Zygosaccharomyces species (supplementary fig. S3).

We subsequently used several approaches to assess the robustness of the HGT hypothesis to explain the phylogenetic incongruences observed. Firstly, we employed a tree reconciliation method based on the duplication/transfer/loss (DTL) model in Notung to evaluate whether the HGT hypothesis was the most parsimonious explanation for the phylogenetic incongruences observed (figs. 4B and 4C). For fixed costs of 1.5 for gene duplications and of 1.0 for gene losses, postulating the occurrence of Event A provides the most parsimonious solution, even when the cost of HGT is set at nine, whereas for Event B the same holds true up to a cost of four for HGT (fig. 4C). These results support the occurrence of HGT in both cases, albeit with stronger substantiation for Event A. The analyses also supported the directionality of the HGT events as proposed in our hypotheses. We also noted that a gene duplication is likely to have taken place in the MRCA of the Eurotiomycetes, followed by unequal loss of paralogs so that one Aspergillus and one Penicillium species still retain two distinct Ffz homologs, which are placed in two distinct sub-clades of the Eurotiales branch of the Ffz tree (fig. 4B and supplementary fig. S2), while other species have lost one of the paralogs. The sub-clade encompassing more genes includes the characterized A. brasiliensis Ffz1 homolog, reported to be functionally similar to those in the Saccharomycotina (Cabral \& Leandro, personal communication), but it is currently unknown whether homologs included in the second sub-clade underwent some form of functional divergence. Two of the four species in the Eurotiales that have lost their Ffz-like transporter, Aspergillus fumigatus and A. clavatus, exhibit some synteny with the FFZ locus of the closely related species Neosartorya fischeri, but while the former species retains a FFZ pseudogene, in the latter the gene is completely absent (supplementary fig. S3). 
The second approach used to consubstantiate the putative HGT events A and B was a comparative topology test, in which the likelihood of the Ffz1 ML-supported topology given by the sequence alignment was compared with the likelihood of alternative topologies in which the Ffz1 tree was constrained to conform to the species phylogeny separately for both events (Fig. 4D). Both SH and AU tests, provided again robust support for the occurrence of Event A but only marginal support for Event B. In order to gather additional evidence that the occurrence of Event $\mathrm{B}$ is the best hypothesis to explain the data, we decided to perform a third analysis focused on this event only. To this end, we examined pairwise distances determined for the concatenated RNA polymerase subunit homolog sequences used to construct the species trees and for Ffz1-like transporter sequences, within the Zygosaccharomyces and the W/S clades as well as between the two clades (fig. 4E). The results showed that while the average pairwise distances recapitulate the phylogenetic distances between the species when RNA polymerase subunits are examined, the outcome is notably different when Ffz1 sequences are used, since average pairwise distances in between clades are in this case very similar to those observed within clades. This suggests that the Ffz1 transporters are more similar between the Zygosaccharomyces and W/S clades than might be expected based on the phylogenetic distance between the two lineages. Hence, this analysis lends further support to the hypothesis that the Zygosaccharomyces clade acquired the FFZ1 gene from a donor in the Wickerhamiella lineage, as depicted in fig. 4B (Event B).

So far, two different biochemically distinct types of Ffz transporters have been identified, Ffz1 and Ffz2. The latter, found to date only in Zygosaccharomyces, is functionally distinct from Ffz1 in that it is capable of transporting both glucose and fructose. The Ffz tree depicted in Fig. 4B suggests that FFZ2 originated from a duplication of an FFZ ancestral gene followed by functional divergence. According to available data, FFZ2 homologs are present in all Zygosaccharomyces species inspected, in addition to one FFZ1 gene, which is consistent with this duplication having occurred in the MRCA of extant Zygosaccharomyces species. To gain additional insight in the evolutionary history of Ffz homologs in Zygosaccharomyces, we examined synteny around the FFZ1 and FFZ2 genes in the three genomes available (supplementary fig. S3). While synteny is conserved around the FFZ1 gene, as might be expected if the HGT event postulated (Event B) involved the MRCA of the genus, no synteny can be discerned in the surroundings of the FFZ2 gene. Hence, no support at the level of gene order conservation was found in this analysis for a common origin of the three FFZ2 genes presently examined.

\section{Functional characterization of novel Ffz-like transporters}

An important question to be answered concerning the evolutionary history of Ffz-like transporters concerns the emergence of their current biochemical function, since the Ffz transporter family is phylogenetically more closely related to Drug: $\mathrm{H}^{+}$antiporters than to other sugar transporters. The 
results obtained for the $U$. maydis transporters depicted in fig. 1 suggest that the transition from drug: $\mathrm{H}^{+}$antiport to sugar transport may have occurred in the MRCA of the Dikarya and leaves the question open of whether Ffz-like transporters in the Pezizomycotina and the Saccharomycotina are largely functionally identical and if not, what is the evolutionary course of events that best explains the biochemical properties of extant transporters. To ascertain this, we first addressed whether substantial functional differences were likely to be found among extant Ffz-like transporters within the widely diverse Pezizomycotina. Hence, we set out to characterize an additional Ffz1 transporter from the Pezizomycotina, chosen from a taxon distantly related to A. brasiliensis, whose Ffz1 homolog has been previously characterized (Cabral \& Leandro, personal communication). To this end, the Fusarium graminearum (Hypocreales) Ffz1-like transporter was selected for expression in the S. cerevisiae hxt-null strain and was found to complement growth of this strain equally well on fructose and mannose, but not on glucose and galactose (fig. 5A). Specific growth rates determined in shake flask cultures with medium containing either fructose or mannose as carbon and energy source confirmed that the $S$. cerevisiae transformant carrying the $F$. graminearum transporter grew vigorously and equally well on fructose and mannose (fig. 5B). Since none of the previously characterized Ffz1 homologs in Ascomycetes was reported to transport mannose, this seemed to constitute a significant functional difference of the $F$. graminearum transporter with respect to other Ffz homologs previously characterized. To assess this, we examined specific growth rates of a $S$. cerevisiae transformant expressing the A. brasiliensis Ffz homolog on both carbon sources and found that similarly to the $F$. graminearum Ffz homolog, it could grow very well on both sugars, although slightly better on fructose (fig. 5B). To find out whether the ability to transport mannose in addition to fructose might have been overlooked when substrate specificity of the various yeast homologs was studied, we reexamined growth on all four hexoses of $S$. cerevisiae transformants expressing the previously characterized Ffz1 homolog from the yeast $Z$. rouxii and also used heterologous expression to functionally characterize the two newly uncovered Ffz-like transporters in Starmerella bacillaris (fig. 5A). We found that all yeast Ffz homologs could also support growth on mannose, although considerably worse than on fructose as judged from specific growth rates measured on both carbon sources (fig. 5B). While Ffz1a from S. bacillaris functionally resembles the $Z$. rouxii transporter, Ffzlb supports much feebler growth on fructose and on solid medium seems to allow for some growth on glucose (fig. 5A), although growth on this sugar could not be detected in liquid medium. To provide insight on the differences in kinetic parameters of fructose transport that might explain poor growth on fructose of transformants harboring Ffz1b, we measured uptake of ${ }^{14} \mathrm{C}$ labeled fructose mediated by either Ffz1a or Ffz1b. The results depicted in fig. 5C, showed for Ffzla the emblematic parameters of Ffz1-like transporters, namely high capacity $\left(\mathrm{V}_{\max }\right)$ and high $\mathrm{K}_{\mathrm{m}}$ values for fructose. Notably, Ffzlb displayed a lower $K_{m}$ and lower $V_{\max }$ than Ffzla, suggesting that the two transporters are not 
functionally identical, the second resembling more closely the Ffz1 transporter from Z. rouxii (Leandro, et al. 2011), which seems to be indispensable for fructophily (Leandro, et al. 2014).

\section{Discussion}

Here we use available fungal genomic data, both publicly available and generated in the course of this work, to elucidate the evolutionary history of Ffz-like fructose transporters, which deserved particular attention for two main reasons. Firstly, evolution of the Ffz1 family seemed to involve relatively recent significant functional shifts, since the closest known relatives of Ffz transporters belong to a Drug: $\mathrm{H}^{+}$antiporter family (DHA1). Secondly, Ffz1 seems to be closely associated with fructophily in yeasts, which is considered a relatively rare but well defined phenotype, relevant among others for the wine industry (Englezos, et al. 2015). This association is anchored on a strict correlation found so far in yeasts between the presence of the transporter and fructophily, as well as on the fact that deletion of the FFZ1 gene abolished fructophily in $Z$. rouxii (Leandro, et al. 2014; Leandro, et al. 2011; Pina, et al. 2004). In addition to genomic data, we employed a strategy previously used successfully to functionally characterize sugar transporters (Young et al. 2014), which consists in evaluating quantitatively their ability to support growth on hexoses of a strain devoid of all endogenous transporters for this type of sugar.

We were able to consubstantiate preliminary phylogenetic analyses (Pina, et al. 2004) suggesting that Ffz transporters do not share the evolutionary origin of any other known sugar transporters. By mining all available genomic data, and generating draft genome sequences for five additional yeast species, we show that Ffz transporters form a clade here named the Ffz-like family, which is distinct from the lineage harboring functionally characterized members of the Drug: $\mathrm{H}^{+}$antiporter family (for example Tpo1 in S. cerevisiae). The sister lineage of the Ffz-like family, includes a large number of proteins of undetermined function. It is therefore unknown whether the first node of the phylogenetic tree in supplementary fig. S1 clearly separates drug antiporters from other carriers. Our data are consistent with the hypothesis that the MRCA of the Ffz-like family has its origin early in the evolution of the Dikarya, because the family has members both from the Ascomycota and from the Basidiomycota, and suggests that this common ancestor was probably a sugar transporter. The strongest evidence for this is that expression of the sole $U$. maydis protein phylogenetically placed within the Ffz-like family showed that it was a broad specificity hexose transporter while the three putative transporters from the same species included in the sister lineage of the Ffz-like family seem to be unable to transport any of the hexoses tested. It is therefore likely that the ability to transport sugars arose only in the MRCA of the Ffz-like family.

Notably, the basidiomycete Ffz-like transporter seems to have a marked preference for glucose, followed by galactose and mannose as substrates. Hence, a pronounced disparity between the 
sugar substrates accepted exists within the Ffz-like family, in particular between the basidiomycete Ffz homolog and its various counterparts in the Ascomycota, which are all capable of transporting fructose and are unable to transport glucose. While studying the sugar specificities of novel Ffz-like transporters, we noted that those from F. graminearum and from S. bacillaris were capable of transporting mannose, in addition to fructose. In view of this, we revisited the biochemical characteristics of the Ffz1 transporter from $Z$. rouxii and found that it also seems to mediate mannose uptake. Since it is a common characteristic of all Ffz homologs studied so far, in both branches of Dikarya, it seems plausible to postulate that this may be an ancestral trait present in the MRCA of the Ffz-like family. Mannose is a quantitatively important component of the fungal cell wall (Bowman and Free 2006; Brul et al. 1997; Klis et al. 2006; Masuoka 2004) being crucial for its integrity, in addition to being a sugar commonly used by fungi as carbon and energy sources, so that mannose uptake is a trait of considerable physiological significance in this group of organisms.

Additional instances of functional differences between $\mathrm{Ffz}$ homologs within the Ascomycota were identified. Firstly, the relative aptitude to transport fructose vs. mannose is not constant among ascomycete Ffz-like transporters, because while the $F$. graminearum homolog seems to take up both sugars equally well, the yeast carriers have a marked bias towards fructose and the A. brasiliensis transporter seems to have an intermediate behavior. Secondly, two instances of duplication followed by functional divergence were discerned. The clearest case concerns a duplication giving rise to the Ffz2-type of transporter that probably occurred in the MRCA of the Zygosaccharomyces genus, because it was present in all species of the genus examined so far. Ffz2-type transporters have higher affinity but lower capacity for fructose transport and are capable of transporting glucose as well. The second duplication was presently uncovered in a single species of the Wickerhamiella/Starmerella (W/S) clade, S. bacillaris. In addition to a gene very similar to FFZ1 from Zygosaccharomyces, named FFZ1a, this species has a second gene, FFZ1b, which supports weaker growth on fructose while resembling the remaining yeast carriers in its ability to support growth on mannose. It is unlikely that deficient growth on fructose is due to some toxic effect of the expression of this protein in S. cerevisiae, because transformants constitutively expressing Ffzlb grow very well on maltose that uses a different uptake system. It is possible that this transporter evolved towards accepting a third, unidentified sugar-like substrate, thereby loosing partly its ability to transport fructose.

While examining both the species distribution and the phylogenetic relationships between Ffz homologs in the Saccharomycotina and the Eurotiales, we found strong evidence for nonvertical acquisition of the gene in at least two significant instances, as well as for many events of gene loss and duplication. The number of reported HGT events in fungi increased rapidly in the past few years, driving the general perception that this mechanism should be recognized as an important provider of genetic innovation in fungi, especially when rapid adaptation to new 
environments is required. The genes transferred in documented cases were very often involved in metabolic innovation, e.g. toxin production or utilization of unusual nutrients (Cheeseman et al. 2014; Greene et al. 2014; Khaldi et al. 2008; Khaldi and Wolfe 2011; Slot and Hibbett 2007; Wisecaver and Rokas 2015), further supporting the link between HGT and the need for swift adaptation. In particular, nutrient transporters form the most abundant functional category among documented cases of HGT in fungi (Coelho, et al. 2013; Marsit, et al. 2015; Richards et al. 2011). The dependence of fungi on osmotrophic nutrition was one reason put forward to explain this (Richards and Talbot 2013). In addition, the relative low connectivity of transporter proteins, which tend to operate in a self-sufficient manner, may also function in favor of successful transfers, because they are more likely to be integrated into a foreign genome and subsequently fixed by selection (Moran et al. 2012). Transporters also stand out when gene loss and duplication events are highlighted in fungal genomes, as well as in various adaptive evolution experiments (Dunn et al. 2013; Payen et al. 2014) and are often located in highly variable portions of the genomes, such as the subtelomeres (Brown, et al. 2010). Altogether, this means that evolution of transporter gene families is usually remarkably dynamic.

The first significant HGT event detected in the present work is likely to have introduced Ffz1 in the Saccharomycotina, thereby setting the stage for evolution of fructophily in yeasts. In order to get more insight in the phylogenetic span of Ffz1 presence in the early-derived W/S lineage, we tested three Wickerhamiella species and found that they were all fructophilic. This indicates that all these species, like W. domercqiae, probably also harbor Ffz1 and that this is likely an ancestral trait in the W/S clade. In addition, we also present strong evidence that the FFZ1 gene was acquired horizontally by the Zygosaccharomyces clade, from a donor in the W/S clade. The occurrence of such an event is also supported by our current knowledge of the ecology of both W/S clade yeasts and Zygosaccharomyces species. Both lineages contain osmotolerant, fructophilic species found in environments with high sugar concentrations. W/S yeasts are very frequently associated to floricolous insects, nectar and honey (Lachance et al. 1998; Lachance et al. 2001; Rosa et al. 2003) where many fructophilic bacteria are also found (Endo and Salminen 2013). It is possible that the acquisition of an FFZI-like gene facilitated the onset of fructophilic metabolism in the common ancestor of the W/S clade. This is supported by the fact that the Ffz1 transporter from A. brasiliensis turned out to be functionally very similar to those found in yeast (Cabral \& Leandro, personal communication). It can be envisaged that the ability to quickly take up and use large amounts of fructose is likely to have had a positive impact on fitness in the fructose-rich floricolous niche. On the other hand, some species of the W/S clade, like Candida stellata and S. bacillaris are frequently isolated from wine fermentations and fruits (Englezos, et al. 2015; Magyar and Toth 2011; Sipiczki 2003). Since Zygosaccharomyces species are often responsible for fermentation of fruit juices and some also occur in honey (Brysch-Herzberg 2004; Cadez et al. 2015; Sinacori et al. 2014; Tofalo, et al. 2009), there seems to be sufficient overlap 
between the ecological niches occupied by Zygosaccharomyces and W/S clade yeasts to facilitate horizontal transfer of genetic material. We favor the hypothesis that this transference occurred from an ancestor of the Wickerhamiella clade to the ancestor of the Zygosaccharomyces genus. Duplication and functional divergence to yield Ffz2 seems, subsequently, to have taken place in the MRCA of the Zygosaccharomyces lineage. This must however be further investigated because we failed to uncover synteny conservation surrounding the FFZ2 locus, as might be expected if the gene had a common origin in the three species examined. Clarification of this aspect must await additional genomic data from other species belonging to the Zygosaccharomyces clade. In the W/S clade only one species carried a tandem duplication of the gene, probably more recent, since the two genes are structurally and functionally less dissimilar.

In summary, using comparative genomics and functional analysis of sugar transporters we traced important steps in the evolution of the Ffz-like family of sugar transporters. This family is monophyletic and seems so far to be composed of sugar transporters, unlike its sister lineage. We propose a model in which a functional switch originated a sugar transporter presumably accepting at least mannose as substrate, after divergence of early-derived fungal lineages, but before the separation of the two phyla in Dikarya. Evolution of Ffz-like transporters in Ascomycota and Basidiomycota may have resulted in substantial functional divergence between Ffz homologs, namely in their ability to transport fructose and glucose. Within the Ascomycota we detected at least two functional variants of the transporter without apparent radical changes in substrate range, but rather with milder alterations in the relative efficiency of uptake of the substrates fructose and mannose. The Saccharomycotina seemed to be originally devoid of Ffzlike transporters and even of carriers belonging to the sister clade of the Ffz-like family, where only other fungal lineages are represented (supplementary fig. S1). Hence, we posit that Ffz-like transporters were introduced in the Saccharomycotina via HGT from a donor in the Eurotiales, a lineage in the Pezizomycotina where we showed that the Ffz homologue from A. brasiliensis supported slightly better growth on fructose than on mannose. We further postulate that this event created an opportunity for the onset of the fructophilic lifestyle in yeasts, possibly impacting up to $10 \%$ of currently known species in the Saccharomycotina concentrated in two lineages, the Zygosaccharomyces genus, that seems to have acquired the fructophilic lifestyle along with the Ffz-like transporter from the early derived W/S yeast lineage through HGT.

\section{Materials and Methods}

\section{Genomic DNA isolation, library preparation and sequencing}

To generate the genome sequences of Starmerella bacillaris PYCC 3044, Candida magnoliae PYCC 2903, Starmerella bombicola PYCC 5882, Wickerhamiella domercqiae PYCC 3067 and Zygosaccharomyces kombuchaensis CBS 8849, high-molecular-weight genomic DNA was 
isolated from yeast cells grown on YPD medium (Coelho, et al. 2013) at $25^{\circ} \mathrm{C}$ for two days using a modified phenol:chloroform:isoamyl alcohol method (Goncalves et al. 2011). DNA was dissolved in TE buffer (10 mM Tris-HCl, $1 \mathrm{mM}$ EDTA, pH 7.6) with RNase A (100 $\mu \mathrm{g} / \mathrm{mL})$. For Illumina sequencing, $1 \mu \mathrm{g}$ of genomic DNA of each strain was used to generate paired-end libraries (300-400 bp insert size) with a manufacturer's kit (TruSeq DNA Prep Kit v2). Each Illumina library was sequenced for 2 x 100 cycles with the Illumina HiSeq2000 system using the services of a commercial provider (University of Wisconsin Biotechnology Center, WI, USA).

\section{Genome assembly and mining for FFZ1 and RNA polymerase genes}

Assembly of about 83, 20, 22, 16 and 11 million Illumina paired-end reads of S. bacillaris, $C$. magnoliae, S. bombicola, W. domercqiae and Z. kombuchaensis, respectively, was carried out with Velvet 1.2.08 (Zerbino and Birney 2008) resulting in average genome coverages of 220x, 54x, 71x, 63x and 23x, respectively. Local BLAST databases were set up for each draft assembly. Ffz homologues and RNA polymerase sequences, used in species tree reconstructions, were retrieved by TBLASTN using the Z. bailii Ffz1 protein sequence (GenBank CAD56485.1) and the $S$. cerevisiae Rpa1, Rpa2, Rpb1, Rpb2, Rpc1 and Rpc2 sequences (GenBank accession numbers P10964.2, P22138.1, P04050.2, P08518.2, P04051.1 and P22276.2, respectively) as queries. Protein coding sequences were predicted using AUGUSTUS (Stanke et al. 2008). Gene sequences used in this study were deposited in GenBank (accession no. KT728676-KT728706).

\section{Initial search for FFZ1 homologues and delimitation of the Ffz1 family}

The initial BLASTP search was performed using the Z. bailii Ffz1 protein sequence as query and using the selected threshold $E$-value threshold of $1 e-60$ retrieved putative homologues from the JGI MycoCosm database (Grigoriev et al. 2014) (as of June 2014). This search yielded a dataset of 1774 protein sequences (Table S1) originating from all lineages in Dikarya and also Mucoromycotina. These sequences were aligned using a fast iterative method (FFT-NS-i) in MAFFT v.6.956 (Katoh and Standley 2014) and subsequently used to reconstruct a NeighborJoining (NJ) guide tree. This dataset was narrowed down to a manageable size by selection of protein sequences representing the main lineages of each subphylum within the Dikarya as main criterion. This dataset was further trimmed through exclusion of Mucoromycotina sequences, none of which clustered with Ffz-like transporters in the guide tree. In addition, previously described Ffz sequences from Z. bailii CLIB 213 (GenBank CAD56485.1 and CDF92071.1) (Pina, et al. 2004) and C. magnoliae JH110 (GenBank AGT55997.1) (Lee, et al. 2014) were included to produce a final dataset composed of 476 sequences (green in Table S1) belonging to 79 fungal species. These sequences were aligned as aforementioned and poorly aligned regions were removed with trimAl v1.2 (Capella-Gutierrez et al. 2009) using the 'gappyout' option. The 
final alignment consisting of 439 positions was analysed in ProtTest v3.2 (Darriba et al. 2011) in order to determine the best model of sequence evolution. A Maximum Likelihood (ML) phylogeny was constructed in RAxML v7.2.8 (Stamatakis 2006) using the PROTGAMMAILG model of amino acid substitution and branch support was determined using 100 rapid bootstraps. Sequences with $E$-values lower than $1 e-137$ and identity higher than $40 \%$ formed a well-defined monophyletic group (highlighted in light yellow, supplementary fig. S1) representing the Ffz-like family. The complete alignment may be found in the Supplementary material.

\section{Screening for the presence of FFZ1 homologues}

To evaluate the prevalence of FFZ1 homologues among fungal genomes, a new BLAST search (BLASTP and TBLASTN) using the Z. bailii Ffz1 protein sequence as query was performed in all fungal species whose genomes were publicly available on JGI MycoCosm and GenBank as of February 2015 (Table S2). BLAST hits with $E$-values lower than the defined threshold of $1 e-137$ and identity higher than $40 \%$ (parameters derived from those observed for the sequences included in the Ffz-like transporter monophyletic group in supplementary fig. S1) and that, moreover, aligned over the majority of their extension were considered to represent positive results. To minimize the chance of scoring false negative results, hits with intermediate $E$-values (between $1 e-137$ and $1 e-100)$ were also phylogenetically analysed to assess their common ancestry with other sequences in the Ffz-like monophyletic group. The pervasiveness of Ffz1 homologues (presence/absence) was calculated for each fungal family (Saccharomycotina) or order (Pezizomycotina) surveyed and plotted in fig. 2. A complete list of fungal genomes inspected for the presence of Ffz1 is given in Table S2.

\section{Species phylogenies}

To reconstruct the phylogenetic relationships among the major lineages within the Ascomycota (fig. 2), three species of each family in the subphylum Saccharomycotina were selected, except in the few cases where genome data was available for only two species (as of February 2015), and for the Wickerhamiella/Starmerella group where all sequenced species were included. In the subphylum Pezizomycotina, three species of each order that comprised more than three genomes publicly available as of February 2015 were chosen and representatives of the earliest-derived subphylum in the Ascomycota (Taphrinomycotina) were also included. Three basidiomycetous species were selected and were used as outgroup. In the reconstruction of the comprehensive species phylogeny in fig. 4A, all species of the Saccharomycotina and Eurotiales (Pezizomycotina) whose genome was available (as of February 2015) were included. Two species of Hypocreales (Fusarium graminearum and Nectria haematococca) as well as two 
Taphrinomycotina species (Saitoella complicata and Schizosaccharomyces pombe) were included, the latter two being used as outgroup. Phylogenetic trees were constructed based on a previously described approach (Coelho, et al. 2013; Parrent, et al. 2009) and using a dataset containing 97 (fig. 2B) and 122 (fig. 4A) concatenated amino acid sequences of six individually aligned and trimmed RNA polymerase subunits (Rpa1, Rpa2, Rpb1, Rpb2, Rpc1 and Rpc2). The resulting alignments, containing 7824 and 9389 positions, respectively, were used to construct rooted ML phylogenies in RAxML v7.2.8 using the PROTGAMMAILG model of amino acid substitution. Branch support for both phylogenetic trees was determined using 100 rapid bootstraps. The complete list of fungal taxa, abbreviated species names, genome databases queried and accession numbers of RNA polymerase proteins is given in Table S3. The complete alignment of each dataset may be found in the Supplementary material.

\section{Phylogenetic analyses of Ffz1-like proteins}

For the restricted dataset in fig. 4B, Ffz1 sequences of all species belonging to both the Saccharomycotina and the Eurotiales were selected. F. graminearum and N. haematococca were used as outgroups. These 51 sequences were aligned using an iterative refinement method (LINS-i) in MAFFT v.6.956 and poorly aligned regions were removed using trimAl software using the 'gappyout' option. The alignment containing 509 amino acids was used to construct a ML phylogeny in RAxML using PROTGAMMAILG model of amino acid substitution. For the extended dataset (Supplementary fig. S2), all Ffz sequences from all the species inspected in Table S2 (represented in green) were included. To these 187 sequences, three proteins from $U$. maydis (UM02585, UM05393 and UM05981) and one protein from Rhodosporidium toruloides (protein ID: 4839) were added as outgroup. These sequences were aligned, trimmed and used to construct a ML phylogeny as described above. Branch support in both trees were assessed by 1000 rapid bootstrap replicates. The complete alignment may be found in the Supplementary material.

\section{Topology and reconciliation analyses}

The likelihood of horizontal gene transfer events were investigated through gene tree-species tree reconciliation analysis using Notung 2.8 (Chen et al. 2000; Stolzer et al. 2012). Duplication and loss costs were kept invariable ( $\mathrm{DC}=1.5$ and $\mathrm{LC}=1.0$ ) while several transfer costs were tested $(\mathrm{TC}=3$ to 11$)$ in order to ascertain the extent at which the two HGT events (A and $\mathrm{B}$ ) were still recovered (figs. 4B and 4C). The approximately unbiased (AU) (Shimodaira 2002) and weighted Shimodaira-Hasegawa (Shimodaira 1998) tests implemented in CONSEL (Shimodaira and Hasegawa 2001) were used to test alternative gene tree topologies (Fig 4D). For both analyses a restricted dataset was used, which included the sequences from all Saccharomycotina and both 
Monascus species but also two other Eurotiales species (A. niger and A. oryzae) and F. versicolor and $F$. oxysporum as outgroups.

\section{Heterologous expression of FFZ1 homologues and closest relatives in S. cerevisiae}

p414TEF and p415TEF plasmids (Mumberg et al. 1995) expressing FFZ homologues from $U$. maydis FB1, S. bacillaris PYCC 3044 and F. graminearum PTF 040 were constructed by homologous recombination in S. cerevisiae EBY.VW4000 as previously described (Coelho, et al. 2013). Final plasmid constructs, primer sequences and specific annealing temperatures are described in Table S4. The pNHA1 plasmid harbouring FFZ1 from Z. rouxii CBS $732^{\mathrm{T}}$ was previously constructed (Leandro, et al. 2011) and was used to transform S. cerevisiae hxt-null EBY.VW4000.

\section{D-[U- $\left.{ }^{14} \mathrm{C}\right]$ fructose uptake assays}

Transformants harbouring FFZ1a and FFZ1b homologues from S. bacillaris PYCC 3044 were grown in liquid YNB medium with $2 \%(\mathrm{w} / \mathrm{v})$ fructose until mid-exponential phase $\left(\mathrm{OD}_{640 \mathrm{~nm}}\right.$ between 0.8 and 1.2), harvested by centrifugation, washed twice with sterile cold water and resuspended to a final concentration of 20 to $22 \mathrm{mg}$ dry weight $/ \mathrm{ml}$. Transport of D-[U- $\left.{ }^{14} \mathrm{C}\right]$ fructose was measured according to previously described procedures (Spencer-Martins and Van Uden 1985). Kinetic parameters were determined by non-linear regression (Michaelis-Menten Equation) using GraphPad Prism (v5.00 for Windows, GraphPad Software, San Diego California USA).

\section{Assessment of glucose and fructose consumption in Wickerhamiella species}

W. domercqiae PYCC 3067, W. domercqiae PYCC 3203, W. australiensis PYCC 6406 and W. cacticola PYCC 6392 were grown overnight in YP medium with $100 \mathrm{~g} \mathrm{~L}^{-1}$ fructose and $100 \mathrm{~g} \mathrm{~L}^{-}$ ${ }^{1}$ glucose at $25^{\circ} \mathrm{C}$ with orbital shaking. Cells were then transferred into the same medium (initial $\mathrm{OD}_{640 \mathrm{~nm}}$ of 0.1 ) and grown under the same conditions. Growth was monitored for 100-200 hours by determining the $\mathrm{OD}_{640 \mathrm{~mm}}$ at different time points. At each time point $2 \mathrm{~mL}$ aliquots of culture were centrifuged and the supernatant was analysed through HPLC. Extracellular concentrations of fructose and glucose $\left(\mathrm{g} \mathrm{L}^{-1}\right)$ were determined by using a carbohydrate analysis column $(300 \mathrm{~mm}$ x 7.8mm, Aminex HPX-87P, Biorad) and a differential refractometer (LKB 2142). Column was kept at $80^{\circ} \mathrm{C}$ and $\mathrm{H}_{2} \mathrm{O}$ was used as the mobile phase at $0.6 \mathrm{~mL} \mathrm{~min}^{-1}$.

\section{Determination of specific growth rates of FFZ1 transformants}

Transformants harbouring FFZ homologues from S. bacillaris (FFZ1a and FFZ1b), Z. rouxii, F. graminearum, U. maydis and A. brasiliensis were grown over night in liquid YNB supplemented as appropriate and containing $2 \%(\mathrm{w} / \mathrm{v})$ of each carbon source (fructose, mannose, glucose and 
galactose). The cells were pre-grown with orbital shaking $(180 \mathrm{rpm})$ at $30^{\circ} \mathrm{C}$ until reaching exponential phase $\left(\mathrm{OD}_{640 \mathrm{~nm}}=0.5-1.2\right)$ and then transferred into the same medium (initial $\mathrm{OD}_{640 \mathrm{~nm}}$ $=0.1$ ) and grown under the same conditions. Growth was monitored by $\mathrm{OD}_{640 \mathrm{~nm}}$ determination until the stationary phase of growth was reached. To determine the specific growth rate $(\mu)$ in fructose and mannose, one transformant was selected for each homologue and three independent assays were performed. The specific growth rate was calculated from four consecutive $\mathrm{OD}_{640 \mathrm{~nm}}$ measurements $\left(\Delta \ln \mathrm{OD}_{640 \mathrm{~nm}} / \Delta \mathrm{t}\right.$, where $\mathrm{t}$ is time).

\section{Strains and growth conditions}

The yeast strains were obtained from PYCC (Portuguese Yeast Culture Collection, Caparica, Portugal). Fusarium graminearum PTF 040 was obtained from Instituto Superior de Agronomia, Universidade de Lisboa, Portugal. All strains were grown and maintained in YPD medium.

\section{Acknowledgments}

We thank Maria José Leandro (LNEG) and Catarina Prista (ISA, UL) for providing plasmid pNHA1-GFP containing FFZ1 from Zygosaccharomyces rouxii and Saccharomyces cerevisiae hxt-null strain harbouring the FFZ1 gene from Aspergillus brasiliensis. This work was supported by grant PTDC/AGR-ALI/112802/2009 and project UID/Multi/04378/2013 from Fundação para

a Ciência e a Tecnologia, Portugal. C.G. and M.A.C. are recipients of grants (SFRH/BD/89489/2012 and SFRH/BPD/79198/2011, respectively) from Fundação para a Ciência e a Tecnologia, Portugal. We also thank the Broad Institute (http://www.broadinstitute.org), and the US Department of Energy Joint Genome Institute (http://www.jgi.doe.gov) for providing access to genomic data produced in collaboration with the user community.

\section{References}

Alarco AM, Balan I, Talibi D, Mainville N, Raymond M. 1997. APl-mediated multidrug resistance in Saccharomyces cerevisiae requires FLRI encoding a transporter of the major facilitator superfamily. J Biol Chem 272:19304-19313.

Barker KS, Pearson MM, Rogers PD. 2003. Identification of genes differentially expressed in association with reduced azole susceptibility in Saccharomyces cerevisiae. J Antimicrob Chemother 51:1131-1140.

Bentley J, Hyatt LS, Ainley K, Parish JH, Herbert RB, White GR. 1993. Cloning and sequence analysis of an Escherichia coli gene conferring bicyclomycin resistance. Gene 127:117-120. 
Bergstrom A, Simpson JT, Salinas F, Barre B, Parts L, Zia A, Nguyen Ba AN, Moses AM, Louis EJ, Mustonen V, et al. 2014. A high-definition view of functional genetic variation from natural yeast genomes. Mol Biol Evol 31:872-888.

Bowman SM, Free SJ. 2006. The structure and synthesis of the fungal cell wall. Bioessays 28:799-808.

Brown CA, Murray AW, Verstrepen KJ. 2010. Rapid Expansion and Functional Divergence of Subtelomeric Gene Families in Yeasts. Curr biol 20:895-903.

Brown CJ, Todd KM, Rosenzweig RF. 1998. Multiple duplications of yeast hexose transport genes in response to selection in a glucose-limited environment. Mol Biol Evol 15:931-942.

Brul S, King A, van der Vaart JM, Chapman J, Klis F, Verrips CT. 1997. The incorporation of mannoproteins in the cell wall of S. cerevisiae and filamentous Ascomycetes. Antonie Van Leeuwenhoek 72:229-237.

Brysch-Herzberg M. 2004. Ecology of yeasts in plant-bumblebee mutualism in Central Europe. FEMS Microbiol Ecol 50:87-100.

Cabral S, Prista C, Loureiro-Dias MC, Leandro MJ. 2015. Occurrence of FFZ genes in yeasts and correlation with fructophilic behavior. Microbiology 10.1099/mic.0.000154.

Cadez N, Fulop L, Dlauchy D, Peter G. 2015. Zygosaccharomyces favi sp. nov., an obligate osmophilic yeast species from bee bread and honey. Antonie Van Leeuwenhoek 107:645-654.

Calabrese D, Bille J, Sanglard D. 2000. A novel multidrug efflux transporter gene of the major facilitator superfamily from Candida albicans (FLU1) conferring resistance to fluconazole. Microbiology 146:2743-2754.

Capella-Gutierrez S, Silla-Martinez JM, Gabaldon T. 2009. trimAl: a tool for automated alignment trimming in large-scale phylogenetic analyses. Bioinformatics 25:1972-1973.

Cheeseman K, Ropars J, Renault P, Dupont J, Gouzy J, Branca A, Abraham AL, Ceppi M, Conseiller E, Debuchy R, et al. 2014. Multiple recent horizontal transfers of a large genomic region in cheese making fungi. Nat Commun 5:2876.

Chen K, Durand D, Farach-Colton M. 2000. NOTUNG: a program for dating gene duplications and optimizing gene family trees. J Comput Biol 7:429-447.

Coelho MA, Goncalves C, Sampaio JP, Goncalves P. 2013. Extensive intra-kingdom horizontal gene transfer converging on a fungal fructose transporter gene. PLoS Genet 9:e1003587.

Darriba D, Taboada GL, Doallo R, Posada D. 2011. ProtTest 3: fast selection of best-fit models of protein evolution. Bioinformatics 27:1164-1165.

Dias PJ, Sa-Correia I. 2013. The drug:H(+) antiporters of family 2 (DHA2), siderophore transporters (ARN) and glutathione:H(+) antiporters (GEX) have a common evolutionary origin in hemiascomycete yeasts. BMC Genomics 14:901.

Diderich JA, Schuurmans JM, Van Gaalen MC, Kruckeberg AL, Van Dam K. 2001. Functional analysis of the hexose transporter homologue HXT5 in Saccharomyces cerevisiae. Yeast 18:1515-1524. 
Dunn B, Paulish T, Stanbery A, Piotrowski J, Koniges G, Kroll E, Louis EJ, Liti G, Sherlock G, Rosenzweig F. 2013. Recurrent rearrangement during adaptive evolution in an interspecific yeast hybrid suggests a model for rapid introgression. PLoS Genet 9:e1003366.

Endo A, Futagawa-Endo Y, Dicks LM. 2009. Isolation and characterization of fructophilic lactic acid bacteria from fructose-rich niches. Syst Appl Microbiol 32:593-600.

Endo A, Salminen S. 2013. Honeybees and beehives are rich sources for fructophilic lactic acid bacteria. Syst Appl Microbiol 36:444-448.

Englezos V, Rantsiou K, Torchio F, Rolle L, Gerbi V, Cocolin L. 2015. Exploitation of the nonSaccharomyces yeast Starmerella bacillaris (synonym Candida zemplinina) in wine fermentation: physiological and molecular characterizations. Int J Food Microbiol 199:33-40.

Feng L, Frommer WB. 2015. Structure and function of SemiSWEET and SWEET sugar transporters. Trends Biochem Sci 40:480-486.

Fitzpatrick DA. 2012. Horizontal gene transfer in fungi. FEMS Microbiol Lett 329:1-8.

Ganapathy-Kanniappan S, Geschwind JF. 2013. Tumor glycolysis as a target for cancer therapy: progress and prospects. Mol Cancer 12:152.

Gold SE, Brogdon SM, Mayorga ME, Kronstad JW. 1997. The Ustilago maydis regulatory subunit of a cAMP-dependent protein kinase is required for gall formation in maize. Plant Cell 9:1585-1594.

Goncalves P, Valerio E, Correia C, de Almeida JM, Sampaio JP. 2011. Evidence for divergent evolution of growth temperature preference in sympatric Saccharomyces species. PLoS One 6:e20739.

Greene GH, McGary KL, Rokas A, Slot JC. 2014. Ecology drives the distribution of specialized tyrosine metabolism modules in fungi. Genome Biol Evol 6:121-132.

Gresham D, Desai MM, Tucker CM, Jenq HT, Pai DA, Ward A, DeSevo CG, Botstein D, Dunham MJ. 2008. The repertoire and dynamics of evolutionary adaptations to controlled nutrient-limited environments in yeast. PLoS Genet 4:e1000303.

Grigoriev IV, Nikitin R, Haridas S, Kuo A, Ohm R, Otillar R, Riley R, Salamov A, Zhao X, Korzeniewski F, et al. 2014. MycoCosm portal: gearing up for 1000 fungal genomes. Nucleic Acids Res 42:D699-704.

Horak J. 2013. Regulations of sugar transporters: insights from yeast. Curr Genet 59:1-31.

Katoh K, Standley DM. 2014. MAFFT: iterative refinement and additional methods. Methods Mol Biol 1079:131-146.

Kellis M, Patterson N, Endrizzi M, Birren B, Lander ES. 2003. Sequencing and comparison of yeast species to identify genes and regulatory elements. Nature 423:241-254.

Khaldi N, Collemare J, Lebrun MH, Wolfe KH. 2008. Evidence for horizontal transfer of a secondary metabolite gene cluster between fungi. Genome Biol 9:R18.

Khaldi N, Wolfe KH. 2011. Evolutionary Origins of the Fumonisin Secondary Metabolite Gene Cluster in Fusarium verticillioides and Aspergillus niger. Int J Evol Biol 2011:423821. 
Klis FM, Boorsma A, De Groot PW. 2006. Cell wall construction in Saccharomyces cerevisiae. Yeast 23:185-202.

Kvitek DJ, Sherlock G. 2011. Reciprocal sign epistasis between frequently experimentally evolved adaptive mutations causes a rugged fitness landscape. PLoS Genet 7:e1002056.

Lachance MA. 2011. Starmerella Rosa \& Lachance In: Kurtzman CP, Fell JW, Boekhout T, editors. The Yeasts, a Taxonomic Study. Amsterdam, Netherlands. p. 811-815.

Lachance MA, Kurtzman CP. 2011. Wickerhamiella van der Walt In: Kurtzman CP, Fell JW, Boekhout T, editors. The Yeasts, a Taxonomic Study. Amsterdam, Netherlands. p. 891-897.

Lachance MA, Rosa CA, Starmer WT, Schlag-Edler B, Barker JS, Bowles JM. 1998.

Wickerhamiella australiensis, Wickerhamiella cacticola, Wickerhamiella occidentalis, Candida drosophilae and Candida lipophila, five new related yeast species from flowers and associated insects. Int J Syst Bacteriol 48:1431-1443.

Lachance MA, Starmer WT, Rosa CA, Bowles JM, Barker JS, Janzen DH. 2001. Biogeography of the yeasts of ephemeral flowers and their insects. FEMS Yeast Res 1:1-8.

Leandro MJ, Cabral S, Prista C, Loureiro-Dias MC, Sychrova H. 2014. The high-capacity specific fructose facilitator $\mathrm{ZrFfz1}$ is essential for the fructophilic behavior of Zygosaccharomyces rouxii CBS 732T. Eukaryot Cell 13:1371-1379.

Leandro MJ, Sychrova H, Prista C, Loureiro-Dias MC. 2011. The osmotolerant fructophilic yeast Zygosaccharomyces rouxii employs two plasma-membrane fructose uptake systems belonging to a new family of yeast sugar transporters. Microbiology 157:601-608.

Lee DH, Kim SJ, Seo JH. 2014. Molecular cloning and characterization of two novel fructosespecific transporters from the osmotolerant and fructophilic yeast Candida magnoliae JH110. Appl Microbiol Biotechnol 98:3569-3578.

Levy S, Kafri M, Carmi M, Barkai N. 2011. The competitive advantage of a dual-transporter system. Science 334:1408-1412.

Libkind D, Hittinger CT, Valerio E, Goncalves C, Dover J, Johnston M, Goncalves P, Sampaio JP. 2011. Microbe domestication and the identification of the wild genetic stock of lagerbrewing yeast. Proc Natl Acad Sci U S A 108:14539-14544.

Lin Z, Li W-H. 2011. Expansion of Hexose Transporter Genes Was Associated with the Evolution of Aerobic Fermentation in Yeasts. Molecular Biology and Evolution 28:131-142.

Magyar I, Toth T. 2011. Comparative evaluation of some oenological properties in wine strains of Candida stellata, Candida zemplinina, Saccharomyces uvarum and Saccharomyces cerevisiae. Food Microbiol 28:94-100.

Marsit S, Mena A, Bigey F, Sauvage FX, Couloux A, Guy J, Legras JL, Barrio E, Dequin S, Galeote V. 2015. Evolutionary Advantage Conferred by an Eukaryote-to-Eukaryote Gene Transfer Event in Wine Yeasts. Mol Biol Evol 32:1695-1707.

Masuoka J. 2004. Surface Glycans of Candida albicans and Other Pathogenic Fungi: Physiological Roles, Clinical Uses, and Experimental Challenges. Clinical Microbiology Reviews 17:281-310.

Moran Y, Fredman D, Szczesny P, Grynberg M, Technau U. 2012. Recurrent Horizontal Transfer of Bacterial Toxin Genes to Eukaryotes. Mol Biol Evol 29:2223-2230. 
Mumberg D, Muller R, Funk M. 1995. Yeast vectors for the controlled expression of heterologous proteins in different genetic backgrounds. Gene 156:119-122.

Pao SS, Paulsen IT, Saier MH. 1998. Major Facilitator Superfamily. Microbiol Mol Biol Rev 62:1-34.

Parrent JL, James TY, Vasaitis R, Taylor AF. 2009. Friend or foe? Evolutionary history of glycoside hydrolase family 32 genes encoding for sucrolytic activity in fungi and its implications for plant-fungal symbioses. BMC Evol Biol 9:148.

Payen C, Di Rienzi SC, Ong GT, Pogachar JL, Sanchez JC, Sunshine AB, Raghuraman MK, Brewer BJ, Dunham MJ. 2014. The dynamics of diverse segmental amplifications in populations of Saccharomyces cerevisiae adapting to strong selection. G3 (Bethesda) 4:399409.

Perez-Ortin JE, Querol A, Puig S, Barrio E. 2002. Molecular characterization of a chromosomal rearrangement involved in the adaptive evolution of yeast strains. Genome Res 12:1533-1539.

Pina C, Goncalves P, Prista C, Loureiro-Dias MC. 2004. Ffz1, a new transporter specific for fructose from Zygosaccharomyces bailii. Microbiology 150:2429-2433.

Reddy VS, Shlykov MA, Castillo R, Sun EI, Saier MH, Jr. 2012. The major facilitator superfamily (MFS) revisited. FEBS $j$ 279:2022-2035.

Reifenberger E, Boles E, Ciriacy M. 1997. Kinetic characterization of individual hexose transporters of Saccharomyces cerevisiae and their relation to the triggering mechanisms of glucose repression. Eur J Biochem 245:324-333.

Reznicek O, Facey SJ, de Waal PP, Teunissen AW, de Bont JA, Nijland JG, Driessen AJ, Hauer B. 2015. Improved xylose uptake in Saccharomyces cerevisiae due to directed evolution of galactose permease Gal2 for sugar co-consumption. J Appl Microbiol 119:99-111.

Richards TA, Leonard G, Soanes DM, Talbot NJ. 2011. Gene transfer into the fungi. Fungal Biol Biotechnol 25:98-110.

Richards TA, Talbot NJ. 2013. Horizontal gene transfer in osmotrophs: playing with public goods. Nat Rev Micro 11:720-727.

Rosa CA, Lachance MA, Silva JO, Teixeira AC, Marini MM, Antonini Y, Martins RP. 2003. Yeast communities associated with stingless bees. FEMS Yeast Res 4:271-275.

Saier MH, Jr., Reddy VS, Tamang DG, Vastermark A. 2014. The transporter classification database. Nucleic Acids Res 42:D251-258.

Shimodaira H. 1998. An Application of Multiple Comparison Techniques to Model Selection. Ann Inst Statist Math 50:1-13.

Shimodaira H. 2002. An approximately unbiased test of phylogenetic tree selection. Syst Biol 51:492-508.

Shimodaira H, Hasegawa M. 2001. CONSEL: for assessing the confidence of phylogenetic tree selection. Bioinformatics 17:1246-1247.

Sinacori M, Francesca N, Alfonzo A, Cruciata M, Sannino C, Settanni L, Moschetti G. 2014. Cultivable microorganisms associated with honeys of different geographical and botanical origin. Food Microbiol 38:284-294. 
Sipiczki M. 2003. Candida zemplinina sp. nov., an osmotolerant and psychrotolerant yeast that ferments sweet botrytized wines. Int J Syst Evol Microbiol 53:2079-2083.

Slot JC, Hibbett DS. 2007. Horizontal transfer of a nitrate assimilation gene cluster and ecological transitions in fungi: a phylogenetic study. PLoS One 2:e1097.

Sousa-Dias S, Gonçalves T, Leyva JS, Peinado JM, Loureiro-Dias MC. 1996. Kinetics and regulation of fructose and glucose transport systems are responsible for fructophily in Zygosaccharomyces bailii. Microbiology 142:1733-1738.

Spencer-Martins I, Van Uden N. 1985. Catabolite interconversion of glucose transport systems in the yeast Candida wickerhamii. BBA-BIOMEMBRANES 812:168-172.

Stamatakis A. 2006. RAxML-VI-HPC: maximum likelihood-based phylogenetic analyses with thousands of taxa and mixed models. Bioinformatics 22:2688-2690.

Stanke M, Diekhans M, Baertsch R, Haussler D. 2008. Using native and syntenically mapped cDNA alignments to improve de novo gene finding. Bioinformatics 24:637-644.

Stolzer M, Lai H, Xu M, Sathaye D, Vernot B, Durand D. 2012. Inferring duplications, losses, transfers and incomplete lineage sorting with nonbinary species trees. Bioinformatics 28:i409i415.

Streubel J, Pesce C, Hutin M, Koebnik R, Boch J, Szurek B. 2013. Five phylogenetically close rice SWEET genes confer TAL effector-mediated susceptibility to Xanthomonas oryzae pv. oryzae. New Phytol 200:808-819.

Tamura K, Stecher G, Peterson D, Filipski A, Kumar S. 2013. MEGA6: Molecular Evolutionary Genetics Analysis version 6.0. Mol Biol Evol 30:2725-2729.

Tofalo R, Chaves-Lopez C, Di Fabio F, Schirone M, Felis GE, Torriani S, Paparella A, Suzzi G. 2009. Molecular identification and osmotolerant profile of wine yeasts that ferment a high sugar grape must. Int J Food Microbiol 130:179-187.

Tomitori H, Kashiwagi K, Asakawa T, Kakinuma Y, Michael AJ, Igarashi K. 2001. Multiple polyamine transport systems on the vacuolar membrane in yeast. Biochem J 353:681-688.

Vidgren V, Multanen JP, Ruohonen L, Londesborough J. 2010. The temperature dependence of maltose transport in ale and lager strains of brewer's yeast. FEMS Yeast Res 10:402-411.

Wieczorke R, Krampe S, Weierstall T, Freidel K, Hollenberg CP, Boles E. 1999. Concurrent knock-out of at least 20 transporter genes is required to block uptake of hexoses in Saccharomyces cerevisiae. FEBS Lett 464:123-128.

Wirsching S, Moran GP, Sullivan DJ, Coleman DC, Morschhauser J. 2001. MDR1-mediated drug resistance in Candida dubliniensis. Antimicrob Agents Chemother 45:3416-3421.

Wisecaver JH, Rokas A. 2015. Fungal metabolic gene clusters-caravans traveling across genomes and environments. Front Microbiol 6:161.

Wright EM, Loo DD, Hirayama BA. 2011. Biology of human sodium glucose transporters. Physiol Rev 91:733-794.

Young EM, Comer AD, Huang H, Alper HS. 2012. A molecular transporter engineering approach to improving xylose catabolism in Saccharomyces cerevisiae. Metab Eng 14:401-411. 
Young EM, Tong A, Bui H, Spofford C, Alper HS. 2014. Rewiring yeast sugar transporter preference through modifying a conserved protein motif. Proc Natl Acad Sci U S A 111:131136.

Yu JH, Lee DH, Oh YJ, Han KC, Ryu YW, Seo JH. 2006. Selective utilization of fructose to glucose by Candida magnoliae, an erythritol producer. Appl Biochem Biotechnol 131:870-879.

Zerbino DR, Birney E. 2008. Velvet: algorithms for de novo short read assembly using de Bruijn graphs. Genome Res 18:821-829.

Zott K, Miot-Sertier C, Claisse O, Lonvaud-Funel A, Masneuf-Pomarede I. 2008. Dynamics and diversity of non-Saccharomyces yeasts during the early stages in winemaking. Int J Food Microbiol 125:197-203.

Figure legends

\section{FIG. 1. Functional analysis of $\boldsymbol{U}$. maydis Ffz homologues expressed as sole hexose} transporters in S. cerevisiae hxt-null. Cells were serially diluted 10-fold (left to right, initial $\left.\mathrm{OD}_{640 \mathrm{~nm}}=0.5\right)$, spotted on solid YNB supplemented with $2 \%(\mathrm{w} / \mathrm{v})$ of glucose, galactose, mannose, fructose and maltose and grown at $30^{\circ} \mathrm{C}$ for four days. EBY.VW4000 cells transformed with p415 TEF were used as control.

FIG. 2. Prevalence of Ffz in Dikarya. (A) Topology of the six Dikarya subphyla and proportion of species encoding a Ffz-like transporter (presence in black, absence in white). (B) Maximum Likelihood phylogeny representing all Saccharomycotina families (three representatives/family) and Pezizomycotina orders (three representatives/order). Percentage of species with and without Ffz homologues is represented for each taxon by the horizontal bar as in (A). Lineages in which Ffz homologues were already characterized are highlighted in different colours. The complete list of genomes inspected is shown in table S2. Number of genomes inspected is shown in brackets in both panels. Bootstrap support values (>75\%) are shown in each tree node as indicated in the key.

FIG. 3. Glucose and fructose consumption profiles of Wickerhamiella species. Cells were grown in liquid YP medium supplemented with $10 \%(\mathrm{w} / \mathrm{v})$ glucose and $10 \%(\mathrm{w} / \mathrm{v})$ fructose. Concentrations of glucose $(\bullet)$ and fructose $(\bullet)$ in the growth media were monitored for 100-200 hours and were determined by HPLC. Cell growth is also shown for the same time period (dashed line). 
FIG. 4. Evidence for HGT in the evolution of the FFZ family. (A) Maximum Likelihood species tree comprising all species for which genome sequences are available in the Saccharomycotina and the Eurotiales. Nectria haematococca and Fusarium graminearum sequences were also included and Saitoella complicata and Schizosaccharomyces pombe (Taphrinomycotina) were used as outgroups. Presence (in black) and absence (in white) of Ffz homologues is shown for each species. Families in which Ffz homologues were characterized are highlighted in colour. Bootstrap support values (>75\%) are shown as indicated in the key. (B) Ffz phylogeny comprising all Ffz protein sequences from the species represented in (A). Postulated HGT events A and B are depicted by arrows. Bootstrap support values ( $>50 \%)$ are shown as indicated in the key. (C) Outcome of gene tree-species tree reconciliation analysis (Notung) for the HGT events indicated in (B). Several transfer costs were tested for each event while duplication and loss costs were maintained constant (at 1.5 and 1.0 respectively). The extent to which the HGT event is recovered is shown in the last column (Yes/No) and cases where more than one equally parsimonious solution is found are marked with (*). (D) Topology analysis (Consel) comparing unconstrained and two differently constrained topologies. Branches representing the different lineages are coloured as in (A) and (B). Topology changes are marked with (*). $P$-values for approximately unbiased (AU) and weighted Shimodaira-Hasegawa (wSH) tests are indicated for each constrained analysis. (E) Pairwise distances (JTT method, 100 bootstraps) were determined in MEGA 6.06 (Tamura et al. 2013) for yeast protein sequences (Ffz1 and concatenated Rpa1-2, Rpb1-2, Rpc1-2). Values were calculated within the W/S and Zygosaccharomyces (Zygo) clades and between the two clades (Interclade) and median values are shown.

FIG. 5. Functional analysis of Ffz homologues expressed as sole hexose transporters in $S$. cerevisiae hxt-null. (A) Cells were serially diluted 10-fold (left to right, initial $\mathrm{OD}_{640 \mathrm{~nm}}=0.5$ ), spotted on solid YNB supplemented with $2 \%(\mathrm{w} / \mathrm{v})$ of glucose, galactose and mannose, and grown at $30^{\circ} \mathrm{C}$ for three days. S. cerevisiae hxt-null cells transformed with $\mathrm{p} 414 \mathrm{TEF}$ were used as control. (B) Specific growth rates for all the transformants grown in liquid YNB medium supplemented with $2 \%(\mathrm{w} / \mathrm{v})$ fructose or $2 \%(\mathrm{w} / \mathrm{v})$ mannose. Statistically significant differences (student's t-test) between growth rates on mannose and on fructose are shown. (C) Estimated $\mathrm{K}_{\mathrm{m}}(\mathrm{mM})$ and $\mathrm{V}_{\max }\left(\mathrm{mmol} \mathrm{h}^{-1} \mathrm{~g}^{-1}\right)$ values for $\mathrm{D}-\left[\mathrm{U}-{ }^{14} \mathrm{C}\right]$ fructose uptake mediated by both S. bacillaris Ffz1 homologues. Species names are abbreviated as in Table S3. 

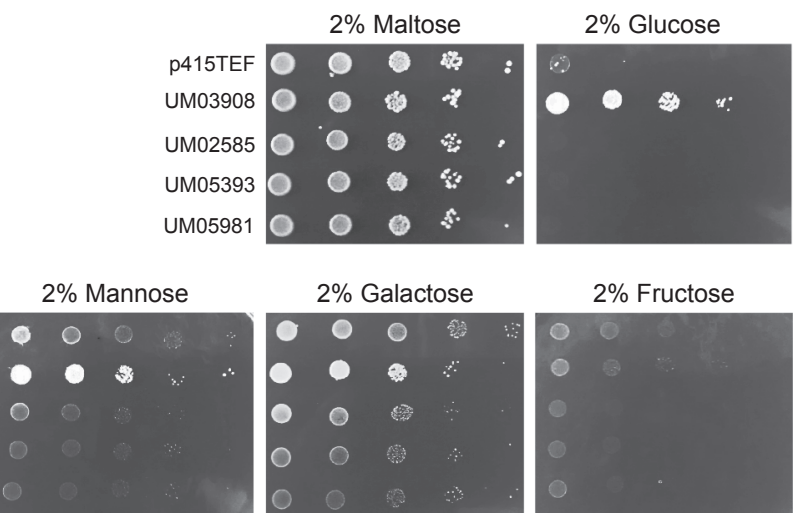
A

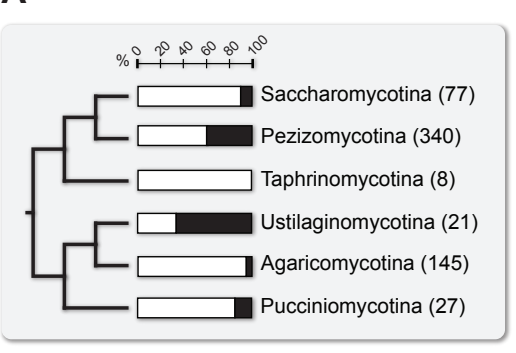

B
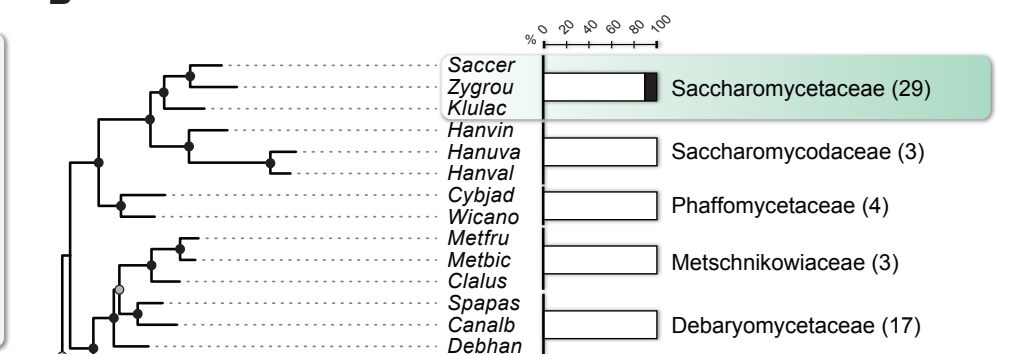

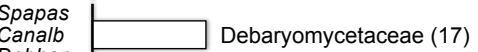

Debhan

Cepalb

Cepfra

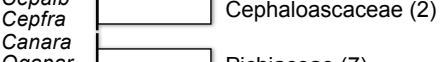

Dekbru

Kompas

Ascrub

Yarlip Dipodascaceae (2)

Sapcla

Blaade

Tripet

Wicdom

Stabac

Canmag
Stabom

Torcas

Torcas

Lipsta

Aspver

Penbil

Theaur

Gymau

Triver

Parbra

Capcor

Exosid

Exooli

Symkoc
Trigui
Xylhev

Wesorn

Aaoarx

Cochet

Aplpru

Botdot

Botdot

Aurpul

Mycpop

Polcit

Polcit

Magpoa

Maegra

Grocla

Sposch

Crypar

Dialon

Valmal

Podans

Mycthe

Mycthe
Verdah
Veralf

Acralc

- Cerman

- Cerfim

Sceapi

- Fusgra

Vallax

Nieexi

Apimon

Eutlat
Dalesc

Erypis

Erynec

Chalon

Chalon

Melvar

Oidmai

Morcon

Terbou

Artoli

Dreste

Monhap

Schjap

Schpom

Rhogra

Psehub

Agabis 

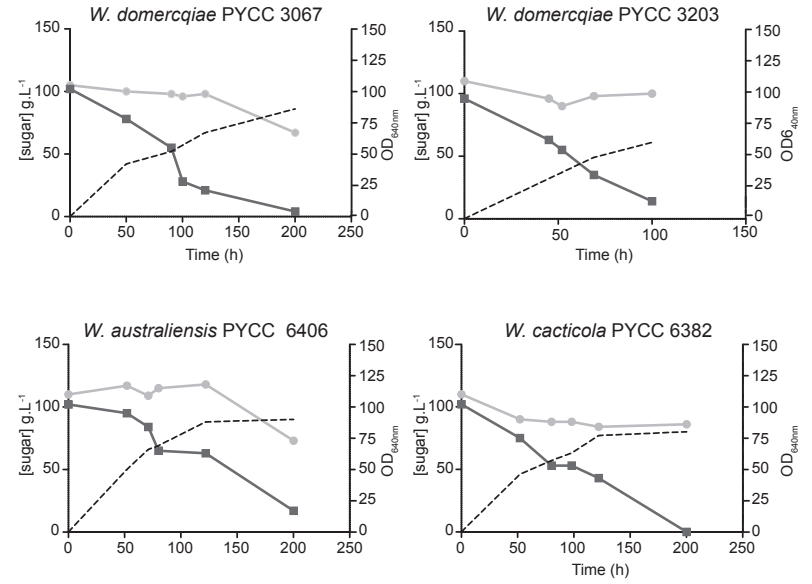

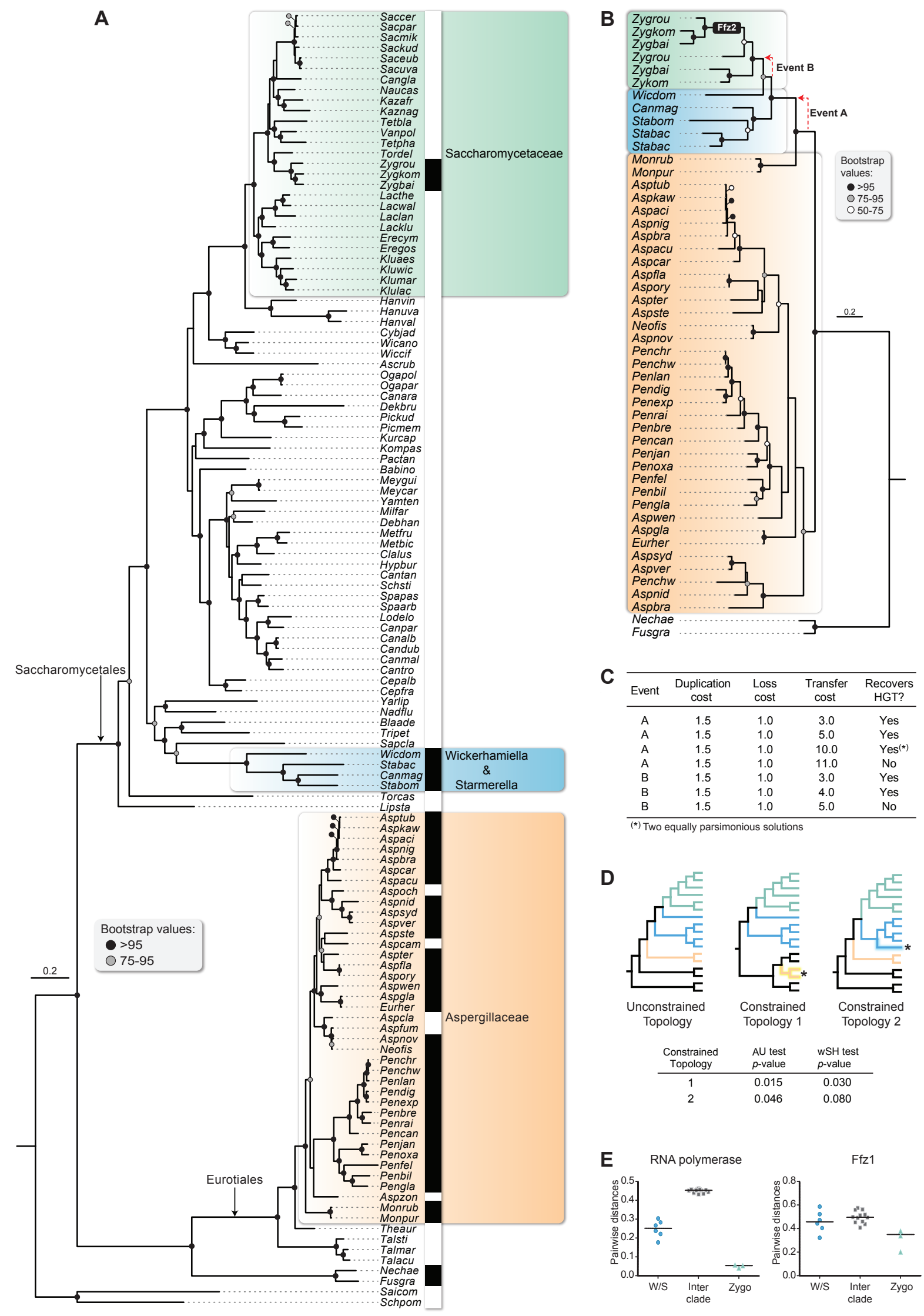

D

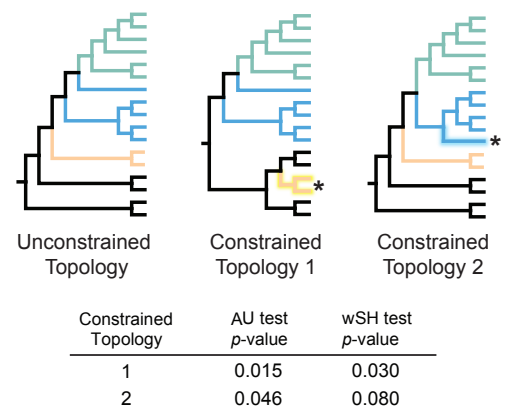

E

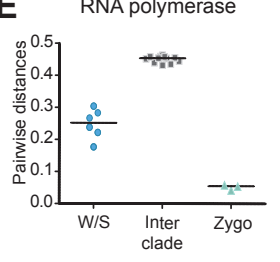



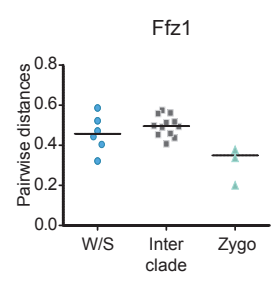




\section{A}

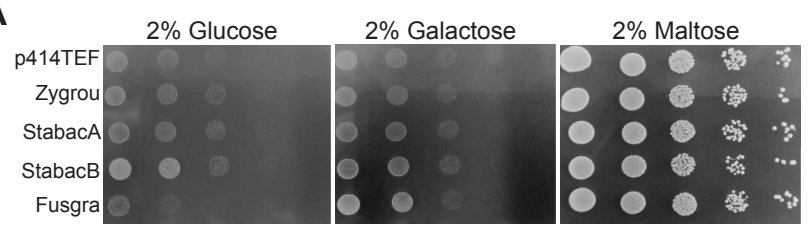

B

\begin{tabular}{|c|c|c|c|}
\hline Homologue & $\mu$ Fructose $\left(h^{-1}\right)$ & $\mu$ Mannose $\left(h^{-1}\right)$ & $\begin{array}{c}\text { Fru vs. Man } \\
(p \text {-value })\end{array}$ \\
\hline ZygrouFfz1 & $0.320 \pm 0.008$ & $0.197 \pm 0.017$ & $<0.0001$ \\
\hline StabacFfz1a & $0.294 \pm 0.005$ & $0.173 \pm 0.007$ & $<0.0001$ \\
\hline StabacFfz1b & $0.121 \pm 0.008$ & $0.201 \pm 0.033$ & $<0.05$ \\
\hline FusgraFfz1 & $0.347 \pm 0.006$ & $0.337 \pm 0.019$ & n.s. \\
\hline AspbraFfz1 & $0.279 \pm 0.012$ & $0.234 \pm 0.013$ & $<0.05$ \\
\hline
\end{tabular}

C

\begin{tabular}{ccc}
\hline Homologue & $\mathbf{V}_{\max }($ Fru $)$ & $\mathbf{K}_{\mathbf{m}}($ Fru $)$ \\
\hline StabacFfz1a & $13.9 \pm 2.6$ & $485.7 \pm 119.3$ \\
StabacFfz1b & $6.7 \pm 0.9$ & $146.8 \pm 38.0$ \\
\hline
\end{tabular}

